Preprint typeset in JHEP style. - HYPER VERSION

CERN-TH/2001-243

December 2001

\title{
Improving the discovery potential of charged Higgs bosons at Tevatron Run 2
}

\author{
Monoranjan Guchait and Stefano Moretti \\ CERN - Theory Division, CH-1211 Geneva 23, Switzerland \\ Emails: monoranjan.guchait@cern.ch,stefano.moretti@cern.ch
}

\begin{abstract}
By exploiting the full process $p \bar{p} \rightarrow t \bar{b} H^{-}$, in place of the standard Monte Carlo procedure of factorising production and decay, $p \bar{p} \rightarrow t \bar{t}$ followed by $\bar{t} \rightarrow \bar{b} H^{-}$, we show how to improve the discovery reach of the Tevatron (Run 2) in charged Higgs boson searches, in the large $\tan \beta$ region. This is achieved in conjunction with dedicated cuts on a 'transverse mass' distribution sensitive to the Higgs boson mass and to 'polarisation' effects in the $H^{-} \rightarrow \tau^{-} \bar{\nu}_{\tau}$ decay channel.
\end{abstract}

KEYwords: Hadronic Colliders, Heavy Quarks Physics, Higgs Physics, Beyond Standard Model. 


\section{Contents}

1. Charged Higgs bosons at the Tevatron 1

2. Charged Higgs boson production in the threshold region 3

3. Signal selection in the $H^{-} \rightarrow \tau^{-} \bar{\nu}_{\tau}$ channel

4. Conclusions 8

\section{Charged Higgs bosons at the Tevatron}

The importance of charged Higgs boson searches at future colliders has in the recent years been emphasised more and more [1]-[3]: the detection of a 'scalar charged' particle would in fact definitely signal the existence of New Physics beyond the Standard Model (SM). Such states are naturally accommodated in non-minimal Higgs scenarios, such as Two-Higgs Doublet Models (2HDMs). A Supersymmetric version of the latter is the Minimal Supersymmetric Standard Model (MSSM) - in fact, a Type II 2HDM with specific relations among neutral and charged Higgs boson masses and couplings, as dictated by Supersymmetry (SUSY) 近.

The Tevatron collider at Fermilab has just begun its second stage of operation, so-called Run 2, with a higher centre-of-mass (CM) energy $(\sqrt{s}=2 \mathrm{TeV})$ and a prospect of collecting something like $15 \mathrm{fb}^{-1}$ of luminosity (per experiment) by the end of its lifetime. This machine will be the first one to probe charged Higgs boson masses in the mass range $M_{H^{ \pm}} \sim m_{t}$ [2]. At present, a lower bound on the charged Higgs boson mass exists from LEP [5], $M_{H^{ \pm}} \gtrsim M_{W^{ \pm}}$, independently of the charged Higgs branching ratios (BRs). This limit is valid within a general $2 \mathrm{HDM}$ whereas, in the low $\tan \beta$ region (say, below 3), an indirect lower limit on $M_{H^{ \pm}}$can be derived in the MSSM from the one on $M_{h^{0}}$ (the mass of the lightest Higgs state of the model): $M_{H^{ \pm}}^{2} \approx M_{W^{ \pm}}^{2}+M_{h^{0}}^{2} \gtrsim(130 \mathrm{GeV})^{2}$.

The main production mode of $H^{ \pm}$scalars at the Tevatron, for $M_{H^{ \pm}}<m_{t}$, is the decay of top (anti)quarks, the latter being produced via QCD in the annihilation of gluon-gluon and quark-antiquark pairs. Simulation studies aiming to assess the discovery reach of the Tevatron in the quest for charged Higgs bosons have relied so far on Monte Carlo (MC) programs, such as PYTHIA [6], HERWIG [7] and ISAJET [8]. Here, the above process is accounted for through the usual procedure 
of factorising the production process, $g g, q \bar{q} \rightarrow t \bar{t}$, times the decay one, $\bar{t} \rightarrow \bar{b} H^{-}$, in the so-called on-shell top approximation,

It is the purpose of this letter to show how this description fails to correctly describe the production and decay phenomenology of charged Higgs bosons when their mass approaches the top one, hence undermining the ability of experimental analyses at Tevatron in pinning down the real nature of these particle (if not detecting them altogether). We will do so by comparing the results obtained in the above approximation with those produced through the full processes $g_{1} g_{2}, q_{1} \bar{q}_{2} \rightarrow t_{3} \bar{b}_{4} H_{5}^{-}$ [9], proceeding via the following graphs:
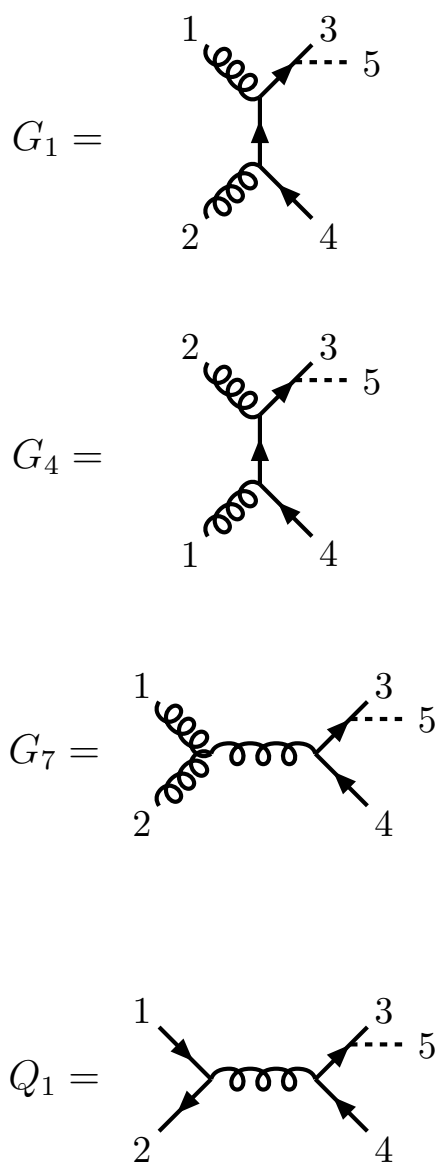
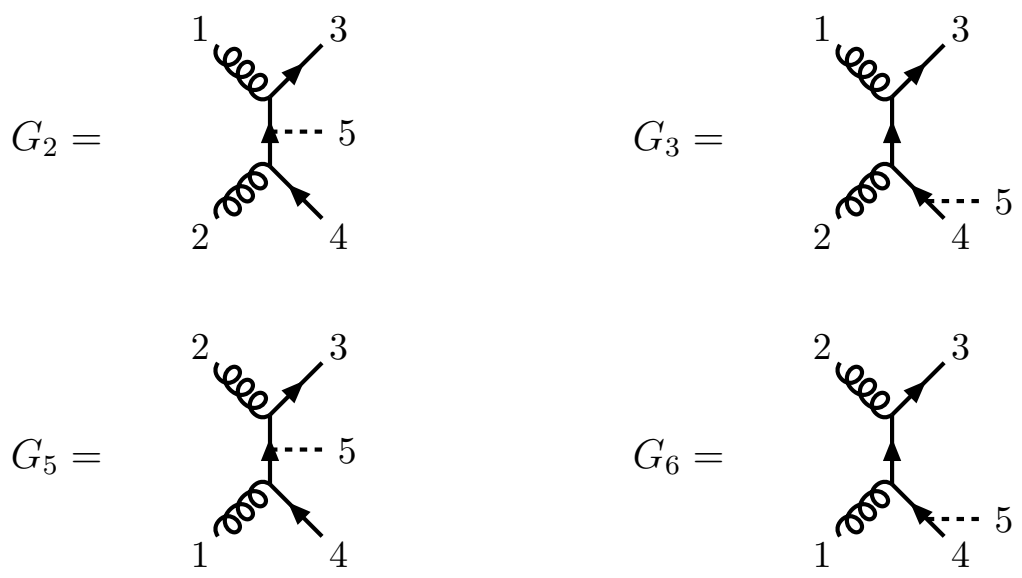

In fact, we will argue the latter being the correct way to describe charged Higgs boson production and decay in the 'threshold region': $M_{H^{ \pm}} \sim m_{t}$. Specifically, we will be exploring Higgs mass values beyond the customary $160 \mathrm{GeV}$ limit considered in Run 2 studies [2], while remaining below $190 \mathrm{GeV}$, where the production cross section is below detection level.

Finally, we will proceed to a signal-to-background analysis, the latter incorporating dedicated selection procedures already advocated in literature, in order to illustrate how the $H^{ \pm}$discovery potential of the Tevatron can be improved, in the 
context of so-called 'direct' (or 'appearance') searches [10. In such a case, specific decay modes of charged Higgs bosons are searched for and kinematical selections are optimised to extract one or another decay signature. In contrast, in 'indirect' (or 'disappearance') searches [11], one employs selection criteria optimised to detect the SM decay of a top (anti)quark, $t \rightarrow b W^{+} \rightarrow b X$, and any loss of such events can be ascribed to the presence of $t \rightarrow b H^{+} \rightarrow b X$ decays. As remarked in [2], the latter method is expected to yield stronger (null) results for integrated luminosities below $2 \mathrm{fb}^{-1}$ or so, whereas with increasing statistics (and, possibly, enhanced detector performances) the former is expected to dominate. Conversely, if a charged Higgs boson exists with mass around $m_{t}$, its presence could be detected through a disappearance search, hence prompting a direct search to confirm discovery.

In this note, among the possible decay signatures of $H^{ \pm}$states, we will concentrate on the $H^{-} \rightarrow \tau^{-} \bar{\nu}_{\tau}$ channel, which is the dominant one for our considered range of $M_{H^{ \pm}}$. The $H^{-} \rightarrow b \bar{t}$ signature originating from $t \bar{b} H^{-}$final states has already been considered (for relatively higher $M_{H^{ \pm}}$values) in Ref. [12]. For a review of typical decay rates of charged Higgs bosons, see Ref. [13].

\section{Charged Higgs boson production in the threshold region}

The subprocesses in (1.1) account for both top-antitop production and decay (graphs $G_{3}, G_{6}$ and $G_{8}$ for gluon-gluon and $Q_{2}$ for quark-antiquark) as well as for Higgsstrahlung (all other graphs) and the relative interferences. In fact, in order to emulate the current implementation in MC programs, one can extract the top-antitop graphs in a gauge invariant fashion, by setting $G_{1}=G_{2}=G_{4}=G_{5}=G_{7}=0$ plus $Q_{1}=0$, and rewriting, in the fixed width scheme, the top propagator as (here, $p=p_{4}+p_{5}$ )

$$
\frac{\not p+m_{t}}{p^{2}-m_{t}^{2}+i m_{t} \Gamma_{t}}\left(\frac{\Gamma_{t}}{\Gamma_{\text {tot }}}\right)^{\frac{1}{2}} \text {. }
$$

When $\Gamma_{t}=\Gamma_{\text {tot }}$, the total top width, the standard expression is recovered. The onshell or Narrow Width Approximation (NWA) can be obtained by taking numerically $\Gamma_{t} \rightarrow 0$, as in this limit eq. (2.1) becomes a representation of the Dirac delta function $\delta\left(p^{2}-m_{t}^{2}\right)$ (apart from a factor $\pi$ ). In practice, the cross section coincides with the one computed as production times BR already for $\Gamma_{t} \sim 10^{-3}$. For the width of the top-quark we have used the tree-level expression, which depends upon both $M_{H^{ \pm}}$ and $\tan \beta$ (the ratio between the vacuum expectation values of the two doublet Higgs fields). Similarly, we have proceeded with the couplings entering the $t \bar{b} H^{-}$vertex.

In Fig. 1, we compare the total cross section obtained by computing processes (1.1) (with $\Gamma_{t}=\Gamma_{\text {tot }}$ ) to the $t \bar{t}$-mediated one in NWA, i.e., $g g, q \bar{q} \rightarrow t \bar{t} \rightarrow t \bar{b} H^{-}$with $\Gamma_{t} \rightarrow 0$. These results are both gauge invariant. For the sake of illustration, we also have included here similar rates obtained by computing the top-antitop diagrams 
only, with $\Gamma_{t}=\Gamma_{\text {tot }}$ (these are subject to a gauge dependence of order $\left.\mathcal{O}\left(\Gamma_{t} / m_{t}\right)\right)^{1}$. Cross sections are shown for two representative $\tan \beta$ values (3 and 30) over the mass range $160 \mathrm{GeV}<M_{H^{ \pm}}<190 \mathrm{GeV}$. However, since the effect that we are investigating is merely kinematical, the same quantitative features would appear for other choices of the former. It is evident how, with $M_{H^{ \pm}}$approaching $m_{t}$, the Higgs boson rates are grossly mis-estimated by the NWA. Hence, it is mandatory to exploit in future MC simulations of $H^{ \pm}$production and decay around the threshold region an implementation based on the $g g, q \bar{q} \rightarrow t \bar{b} H^{-}$matrix elements. Besides, for $M_{H^{ \pm}}>m_{t}-m_{b}$, only the latter can produce a non-zero result. Our default values for $m_{t}$ and $m_{b}$ are 175 and $4.25 \mathrm{GeV}$, respectively, both in the couplings and kinematics ${ }^{2}$.

Also differential distributions can strongly be affected by an approximated modelling of the production process in the threshold region. In Fig. 2, we present the spectra in transverse momentum of the top and bottom quarks, for the full $2 \rightarrow 3$ process and the NWA. Whereas differences in $p_{T}$ are negligible in the case of the Higgs boson (so that this case is not plotted here), they are sizable for the top and dramatic for the bottom quark. Whereas one should expect the impact of the differences seen in the top quark distribution to eventually be marginal, owning to the fact that this particle is actually unstable and that its three-body decay products are subject to the (cumulative) effect of usual detector resolution uncertainties, this is no longer true for the bottom quark, which fragments directly into hadrons. Besides, the availability of the newly implanted silicon vertex detector may render the tagging of $b$-quarks a crucial ingredient in detection strategies of charged Higgs bosons at Run 2. Results in Fig. 2 are shown for $M_{H^{ \pm}}=170 \mathrm{GeV}$ and $\tan \beta=3$. Whereas the described effects are insensitive to the actual value of the latter, a difference choice of the former can modify the relative shape of the two curves (full and NWA) significantly, but the distinctive features seen here remain qualitatively the same for any choice of $M_{H^{ \pm}}$in the considered mass interval.

Before moving on to the decay analysis, one final remark is in order concerning the production stage. In Fig. 1, we also have presented the cross sections of the so-called 'Drell-Yan mode', $\left.q \bar{q} \rightarrow H^{+} H^{-}[1]\right]^{3}$, followed by $H^{+} \rightarrow t \bar{b}$. This is the

\footnotetext{
${ }^{1}$ They are meant to illustrate what portion of the difference between the full results and those in NWA is due to finite width effects of the top-quark, the remainder of it coming from the contribution of the other diagrams and the relative interferences.

${ }^{2}$ When we will discuss decay rates of the charged Higgs boson, the two masses above will be the input values of HDECAY [14] - the package that we used for our numerical estimates.

${ }^{3}$ In fact, this nomenclature is somewhat misleading, as $b \bar{b} \rightarrow H^{+} H^{-}$contributions proceeding via double Higgs-strahlung or neutral Higgs boson mediation (e.g., $h^{0}, H^{0}$ and $A^{0}$ in the MSSM), as opposed to gauge boson exchange, i.e., $q \bar{q} \rightarrow \gamma^{*}, Z^{*} \rightarrow H^{+} H^{-}$, are not entirely negligible, particularly at the LHC, where they are in fact dominant at large $\tan \beta$ - similarly for the loopinduced contributions, $g g \rightarrow H^{+} H^{-}$[16]. (Notice that we include all such subprocesses in our calculation.)
} 
only process competing with $g g, q \bar{q} \rightarrow t \bar{b} H^{-}$at Tevatron energies, at least in the low to intermediate $\tan \beta$ region, say, $1 \lesssim \tan \beta \lesssim 10$. (At the Large Hadron Collider (LHC), many more production modes exist [17].) In principle then, one should also investigate the $q \bar{q} \rightarrow H^{+} H^{-} \rightarrow t \bar{b} H^{-}$channel, alongside $g g, q \bar{q} \rightarrow t \bar{b} H^{-}$in (1.1). In practice, though, in direct investigations of the $H^{-} \rightarrow \tau^{-} \bar{\nu}_{\tau}$ decay channel, one is implicitly concerned with large $\tan \beta$ values only. The reason is twofold. On the one hand, an appearance search in the threshold region with a very low $\tan \beta$ (say, below 1.5) would have to be based on $H^{-} \rightarrow s \bar{c}$ decays, which are very challenging because of an overwhelming QCD noise; whereas for $\tan \beta=2-3$ one finds that $H^{ \pm} \rightarrow W^{ \pm(*)} h^{0}$ decays can be relevant (see 18 for some LHC studies), and these are strongly model dependent (e.g., in the MSSM they are no longer viable, given the recent limits on $M_{h^{0}}$ from LEP in this scenario). To date, CDF [10] has only published results for $H^{-} \rightarrow \tau^{-} \bar{\nu}_{\tau}$, which are valid for $\tan \beta \gtrsim 4$. On the other hand, for $\tan \beta$ close to $\sqrt{m_{t} / m_{b}}$, the strength of the $t \bar{b} H^{-}$coupling - entering the diagrams in (1.1) - reaches a minimum, in the end rendering the production cross section unobservable. This basically occurs over the interval $4 \lesssim \tan \beta \lesssim 10$. Besides, for $M_{H^{ \pm}} \sim m_{t}$ and $\tan \beta \gtrsim 10$, the $H^{-} \rightarrow \tau^{-} \bar{\nu}_{\tau}$ decay mode is truly dominant, as one can appreciate by combining the production cross sections of Fig. 1 with typical $H^{ \pm}$ decay rates [13]. This is made clear in the left-hand side of Fig. 3, for a general Type II 2HDM (note the relevance of $H^{-} \rightarrow b \bar{t}^{(*)}$ off-shell decays at low $\tan \beta$ even well below $\left.M_{H^{ \pm}}=m_{t}+m_{b}\right)$. The statement remains true also in the case of charged Higgs boson decays into Supersymmetric particles [19], as could well happen in the MSSM (according to current experimental limits). For example, in the right-hand side of Fig. 3, we display the corresponding $\sigma \times$ BR rates in the MSSM with $M_{2}=130 \mathrm{GeV}$ and $\mu=300 \mathrm{GeV}$, yielding: $M_{\tilde{\chi}_{1,2}^{ \pm}} \approx 330,106 \mathrm{GeV}$ and $M_{\tilde{\chi}_{1,2,3,4}^{ \pm}} \approx 58,109,304,331$ $\mathrm{GeV}$ (for $\tan \beta=3$ ); $M_{\tilde{\chi}_{1,2}^{ \pm}} \approx 118,326 \mathrm{GeV}$ and $M_{\tilde{\chi}_{1,2,3,4}^{ \pm}} \approx 63,119,309,322 \mathrm{GeV}$ (for $\tan \beta=30$ ). Here, $M_{\tilde{\chi}_{i}^{ \pm}}$and $M_{\tilde{\chi}_{j}^{0}}$ are the two $(i=1,2)$ chargino and four $(j=1,2,3,4)$ neutralino masses, respectively. In fact, the dominant decays into Supersymmetric particles of $H^{ \pm}$bosons are those into chargino-neutralino pairs, since typically one has $M_{H^{ \pm}}>M_{\tilde{\chi}_{i}^{ \pm}}+M_{\tilde{\chi}_{j}^{0}}$ for some $i j$ combination (see Ref. [20], where more plots along the same lines can also be found). We defer the detailed investigation of the low to intermediate $\tan \beta$ interval to [21].

\section{Signal selection in the $H^{-} \rightarrow \tau^{-} \bar{\nu}_{\tau}$ channel}

The signature of interest here is $p \bar{p} \rightarrow t \bar{b} H^{-}$, followed by $H^{-} \rightarrow \tau^{-} \bar{\nu}_{\tau}$, with the top quark decaying hadronically, $t \rightarrow b q \bar{q}^{\prime}$. The same type of event topologies may appear in the SM process $p \bar{p} \rightarrow t \bar{b} W^{-} \rightarrow t \bar{b} \tau^{-} \bar{\nu}_{\tau}$, which is in fact the dominant irreducible background. This should be clear, if one notices that one of the subprocesses entering the background is $p \bar{p} \rightarrow t \bar{t} \rightarrow t \bar{b} W^{-}$, i.e., top-antitop production and decay in the 
$\mathrm{SM}$, for which one has $\sigma(p \bar{p} \rightarrow t \bar{t}) \sim 7-8 \mathrm{pb}$ at Tevatron for $\sqrt{s}=2 \mathrm{TeV}$ (in our calculation, the full set of tree-level diagrams leading to $t \bar{b} W^{-}$final states has been computed).

The $\tau$ 's can be tagged as narrow jets in their 'one-prong' hadronic decay modes, which represent $90 \%$ of the hadronic decay rate and about $50 \%$ of the total one. The main components of such decays are: $\tau^{ \pm} \rightarrow \pi^{ \pm} \nu_{\tau}(12.5 \%), \rho^{ \pm} \nu_{\tau}(24 \%)$ and $a_{1}^{ \pm} \nu_{\tau}(7.5 \%)$, with in turn $\rho^{ \pm} \rightarrow \pi^{ \pm} \pi^{0}$ and $a_{1}^{ \pm} \rightarrow \pi^{ \pm} \pi^{0} \pi^{0}$. This distinguishing feature is in contrast to the typical appearance of quark- and gluon-jets, which yield 'multi-prong' hadronic topologies in the detectors. This characteristic difference can profitably be exploited to efficiently isolate the hadronic $\tau$-signals from QCD backgrounds of the form $W^{ \pm}+$jets and $Z^{0}+$ jets, which we have then ignored here.

We have studied our signal and background processes using a very simple partonlevel MC analysis, i.e., without taking into account fragmentation effects of partons. In our numerical calculation we have set the renormalisation and factorisation scales equal to the partonic CM energy, $Q^{2}=\hat{s}$, and the CTEQ4M 22 Parton Distribution Functions were used throughout. For the selection of events, we have adopted the following set of cuts in transverse momentum, $p_{T}$, and pseudorapidity, $\eta$, as well as transverse missing momentum, $\not p_{T}$.

1. Tau-jets are selected if they satisfy the following criteria: $p_{T}^{\tau}>15 \mathrm{GeV}$ and $\left|\eta^{\tau}\right|<2.5$

2. We require $\not p_{T}>20 \mathrm{GeV}$, since the presence of neutrinos from $H^{-}$decays and invisible decay products of $\tau^{\prime}$ 's (mainly $\pi^{0}$ 's) implies that a significant fraction of transverse momentum goes undetected.

3. Quark-jets are selected by imposing $p_{T}^{j}>20 \mathrm{GeV}$ and $\left|\eta^{j}\right|<2.5$. We require at least one of these to be tagged as a $b$-jet.

4. We demand that two un-tagged jets have an invariant mass around $M_{W^{ \pm}}$, e.g., $\left|M_{q \bar{q}^{\prime}}-M_{W^{ \pm}}\right|<10 \mathrm{GeV}$ and that the $b$-jet in combination with other two un-tagged jets produces an invariant mass close to $m_{t}$, e.g., $\left|M_{b q \bar{q}^{\prime}}-m_{t}\right|<15$ $\mathrm{GeV}$.

After the implementation of these cuts, we have found that the cross section for the signal is, e.g., 0.6(5.5) fb for $M_{H^{ \pm}}=170 \mathrm{GeV}$ and $\tan \beta=3(40)$; whereas for the background process one has $90 \mathrm{fb}$. Clearly, signal-to-background ratios $(S / B$ 's) of this sort are insufficient to establish the presence of $H^{ \pm}$states. Hence, further cuts have to be devised.

To this end, we have exploited another kinematic variable: a transverse mass, $M_{T}$, constructed from the visible $\tau$-jet and the missing energy, i.e.,

$$
M_{T}=\sqrt{2 p_{T}^{\tau} p_{T}(1-\cos \Delta \phi)},
$$


as introduced in Ref. [23]. In the case of the signal, the $\tau$-jets are heavily boosted relatively to the case of the background, as the charged Higgs masses considered here are much heavier than $M_{W^{ \pm}}$. This leads to a backward(forward) peak in the azimuthal angle distribution, $\Delta \phi$, identified by the directions of the $\tau$-jet and the missing momentum in the signal(background): see Fig. 4 . By imposing $M_{T}>M_{W^{ \pm}} \approx 80 \mathrm{GeV}$, the background is reduced by more than two orders of magnitude, while the signal cross section is suppressed to a much lesser extent. For example, for $M_{H^{ \pm}}=170$ $\mathrm{GeV}$, the latter becomes $0.4(3.5) \mathrm{fb}$ for $\tan \beta=3(40)$ while the former comes down to a manageable $0.22 \mathrm{fb}$. In Tab. 1 we summarise the signal and background cross sections for some representative values of $M_{H^{ \pm}}$and $\tan \beta$, after all cuts described above.

Before converting the numbers in Tab. 1 into event rates and significances, one has to take into account the finite efficiency of the detectors in particle identification. For example, $\tau$-identification efficiencies are estimated to be of order $50 \%$ [24], similarly for the tagging of any $b$-jet [2]. Hence, one should more realistically expect both signal and background rates to be further reduced by a factor of 4 or so. In the end, however, the chances of extracting the $H^{ \pm} \rightarrow \tau^{ \pm} \nu_{\tau}$ signal after $15 \mathrm{fb}^{-1}$ of luminosity are rather good, at least at large $\tan \beta$, while being negligible at low to intermediate values of the latter (as already argumented). Notice that this remains true for charged Higgs masses above $m_{t}$ as well, say, up to $180 \mathrm{GeV}$ or so, where a handful of signal events should survive in each experiment.

This situation is rather encouraging, especially considering that there may be some room to further improve the $S / B$ 's if one recalls that the distributions of oneprong hadronic decay tracks of $\tau$ 's are strongly sensitive to the polarisation state of the lepton (see, e.g., Refs. [25, 26] for a detailed discussion). Basically, the key feature relevant to our purposes is the correlation between the latter and the energy sharing among the decay pions. In fact, it is to be noted that the spin state of $\tau^{\prime}$ s coming from $H^{ \pm}$- and $W^{ \pm}$-boson decays are opposite: i.e., $H^{-} \rightarrow \tau_{R}^{-} \bar{\nu}_{R}$ and $H^{+} \rightarrow \tau_{L}^{+} \nu_{L}$ whereas $W^{-} \rightarrow \tau_{L}^{-} \bar{\nu}_{R}$ and $W^{+} \rightarrow \tau_{R}^{+} \nu_{L}$ (neglecting leptonic mass effects, as we did here). Ultimately, this leads to a significantly harder momentum distribution of charged pions from $\tau$-decays for the $H^{ \pm}$-signal compared to the $W^{ \pm}$-background, which can then be exploited to increase $S / B$. This is true for the case of one-prong decays into both $\pi^{ \pm}$'s and longitudinal vector mesons, while the transverse component of the latter dilutes the effect and must be somehow eliminated. This can be done inclusively, i.e., without having to identify the individual mesonic component of the one-prong hadronic topology. In doing so [27, we will closely follow Ref. 26].

The mentioned transverse components of the signal as well as those of the background can adequately be suppressed by requiring that $80 \%$ of the $\tau$-jet (transverse) 
energy is carried away by the $\pi^{ \pm}$'s, i.e.:

$$
R=\frac{p^{\pi^{ \pm}}}{p_{T}^{\tau}}>0.8 .
$$

The enforcement of this constraint reduces by a factor of 5 the background, while costing to the signal a 50\% suppression (for any relevant charged Higgs mass).

Incidentally, we should mention that acceptance efficiencies for the selection procedure that we have chosen here are very similar in the case of the signal for both the $2 \rightarrow 3$ simulation and the NWA. However, this should not be surprising, as we have imposed no requirement of a second $b$-tag. In fact, in most cases, only one $b$-quark enters the detector region - the one produced in the (hadronic) decay of the top quark in the $t \bar{b} H^{-}$final state. In contrast, things would be rather different if two $b$-tags were asked, both at $p_{T}^{b}>20 \mathrm{GeV}$, as it should be clear from the right-hand side plot in Fig. 2 .

\section{Conclusions}

In summary, we have demonstrated that concrete prospects exist at Tevatron Run 2 of extending the discovery reach of charged Higgs bosons up to masses of order $m_{t}$, in the large $\tan \beta$ region, in the context of direct searches. This can be achieved by combining the following ingredients.

- To emulate the production of charged Higgs boson events by resorting to the full $g g, q \bar{q} \rightarrow t \bar{b} H^{-}$process, as opposed to the traditional procedure of generating the scalar particles in on-shell top decays, from $g g, q \bar{q} \rightarrow t \bar{t}$ events. In fact, the former not only includes the dynamics of the latter, but also embeds charged Higgs production from Higgs-strahlung and relative interferences.

- To search for 'one-prong' hadronic decays of $\tau$-leptons produced in $H^{-} \rightarrow$ $\tau^{-} \bar{\nu}_{\tau}$ events, in presence of a single $b$-tag, usual detector requirements and after $W^{ \pm}$- and $t$-mass reconstruction in the accompanying hadronic system, $t \rightarrow b W^{+} \rightarrow$ jets.

- To enforce a cut in a typical transverse mass $\left(M_{T}\right)$ distribution, which is bound to assume values below the particle yielding the $\tau$-leptons $\left(H^{ \pm}\right.$for the signal and $W^{ \pm}$for the background). Besides, since a cut as low as $M_{T}>M_{W^{ \pm}}$is sufficient to reduce the $W^{ \pm}$background to negligible levels, the same distribution can also be used to eventually fit the unknown charged Higgs boson mass, when $M_{H^{ \pm}} \sim m_{t}$.

- Finally, to exploit well-known polarisation effects in the case of $\tau^{ \pm} \rightarrow \pi^{ \pm} \nu_{\tau}$, $\rho^{ \pm} \nu_{\tau}$ and $a_{1}^{ \pm} \nu_{\tau}$ decays. 
In drawing our conclusions, we have relied on a parton-level analysis. However, we expect that its main features should remain valid even in presence of fragmentation/hadronisation effects. We do advocate a selection procedure along the above lines to be investigated at a more phenomenological level (including realistic detector simulations) by the Tevatron experiments. For example, the mentioned $2 \rightarrow 3$ description of the $H^{ \pm}$production dynamics is available since version 6.3 in the HERWIG event generator [28] while polarised $\tau$-decays are now implemented in version 6.4 29] (also including an interface to TAUOLA [30]).

In order to motivate such analyses, we propose a benchmark scenario that may eventually emerge from these. In Fig. 5, we present the exclusion regions, below the level curves, in the $\tan \beta-M_{H^{ \pm}}$plane that can potentially be explored at $95 \%$ confidence level (CL) at the upgraded Tevatron, for two luminosity options, 5 and 15 $\mathrm{fb}^{-1}$, by using the tools and the strategy outlined here. The significances $\sigma \equiv S / \sqrt{B}$ used for the contours in Fig. 5 have been estimated at the parton level, after the sequence of cuts in 1.-4. and the one in transverse mass, $M_{T}>M_{W^{ \pm}}$, hence before the one in (3.2) and without including the mentioned efficiencies. Incomplete as these estimates might be at this stage, it is clear the dramatic improvement (both in $\tan \beta$ and $M_{H^{ \pm}}$reach) that could be achieved, if one compares our plot to Fig. 102 of Ref. [2].

\section{Acknowledgements}

MG is thankful to the High Energy Group at the Abdus Salam International Centre for Theoretical Physics for hospitality during the final phase of this work.

\section{References}

[1] M. Carena, J.S. Conway, H.E. Haber and J.D. Hobbs (conveners), Proceedings of the 'Tevatron Run II SUSY/Higgs' Workshop, Fermilab, Batavia, Illinois, USA, February-November 1998, preprint Fermilab-Conf-00/279-T and SCIPP-00/37, October 2000, hep-ph/0010338.

[2] D. Chakraborty, in Ref. [1].

[3] K.A. Assamagan, A. Djouadi, M. Drees, M. Guchait, R. Kinnunen, J.-L. Kneur, D.J. Miller, S. Moretti, K. Odagiri and D.P. Roy, in Proceedings of the Workshop 'Physics at TeV Colliders', Les Houches, France, 8-18 June 1999, preprint January 2000, hep-ph/0002258.

[4] J.F. Gunion, H.E. Haber, G.L. Kane and S. Dawson, "The Higgs Hunters' Guide" (Addison-Wesley, Reading, MA, 1990). 
[5] See, e.g.: M. Antonelli and S. Moretti, in Proceedings of the '13th Convegno sulla Fisica al LEP (LEPTRE 2001)', Rome, Italy, 18-20 April 2001, preprint CERN$\mathrm{TH} / 2001-152$, June 2001, hep-ph/0106332 (and references therein).

[6] T. Sjöstrand, Comp. Phys. Comm. 82 (1994) 74; T. Sjöstrand, P. Edén, C. Friberg, L. Lönnblad, G. Miu, S. Mrenna and E. Norrbin, Comp. Phys. Commun. 135 (2001) 238; T. Sjöstrand, L. Lönnblad and S. Mrenna, preprint LU TP 01-21, August 2001, hep-ph/0108264.

[7] G. Marchesini, B.R. Webber, G. Abbiendi, I.G. Knowles, M.H. Seymour and L. Stanco, Comp. Phys. Commun. 67 (1992) 465; G. Corcella, I.G. Knowles, G. Marchesini, S. Moretti, K. Odagiri, P. Richardson, M.H. Seymour and B.R. Webber, preprint Cavendish-HEP-99/17, December 1999, hep-ph/9912396; JHEP 0101 (2001) 010.

[8] H. Baer, F.E. Paige, S.D. Protopopescu and X. Tata, preprint BNL-HET-99-43, FSU-HEP-991218, UH-511-952-00, December 1999, hep-ph/0001086 (and references therein).

[9] J.F. Gunion, Phys. Lett. B322 (1994) 125; J.L. Diaz-Cruz and O.A. Sampayo, Phys. Rev. D50 (1994) 6820; D.J. Miller, S. Moretti, D.P. Roy and W.J. Stirling, Phys. Rev. D61 (2000) 055011; S. Moretti and D.P. Roy, Phys. Lett. B470 (1999) 209; F. Borzumati, J.-L. Kneur and N. Polonsky, Phys. Rev. D60 (1999) 115011.

[10] CDF Collaboration: F. Abe et al., Phys. Rev. Lett. 79 (1997) 357; D0 Collaboration: T. Affolder et al., Phys. Rev. D62 (2000) 012004.

[11] D0 Collaboration: B. Abbott et al., Phys. Rev. Lett. 82 (1999) 4975; D. Chakraborty (on behalf of the D0 and CDF Collaborations), presented at the '13th Hadron Collider Physics Conference', preprint FERMILAB-Conf-99/143-E.

[12] A. Belyaev, D. Garcia, J. Guasch and J. Solá, preprint FSU-HEP-050101, KA-TP15-2001, UAB-FT-512, May 2001, hep-ph/0105053.

[13] S. Moretti and W.J. Stirling, Phys. Lett. B347 (1995) 291; Erratum, ibidem B366 (1996) 451; A. Djouadi, J. Kalinowski and P.M. Zerwas, Z. Phys. C70 (1996) 435; E. Ma, D.P. Roy and J. Wudka, Phys. Rev. Lett. 80 (1998) 1162.

[14] A. Djouadi, J. Kalinowski and M. Spira, Comput. Phys. Commun. 108 (1998) 56.

[15] E. Eichten, I. Hinchliffe, K. Lane and C. Quigg, Rev. Mod. Phys. 56 (1984) 579.

[16] S.S.D. Willenbrock, Phys. Rev. D35 (1987) 173.

[17] J.F. Gunion, H.E. Haber, F.E. Paige, W.-K. Tung and S.S.D. Willenbrock, Nucl. Phys. B294 (1987) 621; S. Moretti and K. Odagiri, Phys. Rev. D55 (1997) 5627; S.S.D. Willenbrock, in Ref. [16]; A.A. Barrientos Bendezú and B.A. Kniehl, Nucl. Phys. B568 (2000) 305; A. Krause, T. Plehn, M. Spira and P.M. Zerwas, Nucl. 
Phys. B519 (1998) 85; Y. Jiang, W.-G. Ma, L. Han, M. Han and Z.-H. Yu, J. Phys. G23 (1997) 385; Erratum, ibidem G23 (1997) 1151; O. Brein and W. Hollik, Eur. Phys. J. C13 (2000) 175; Y. Jiang, W.-G. Ma, L. Han, M. Han and Z.-H. Yu, J. Phys. G24 (1998) 83; D.A. Dicus, J.L. Hewett, C. Kao and T.G. Rizzo, Phys. Rev. D40 (1989) 787; A.A. Barrientos Bendezú and B.A. Kniehl, Phys. Rev. D59 (1999) 015009; ibidem D61 (2000) 097701; ibidem D63 (2001) 015009; S. Moretti and K. Odagiri, Phys. Rev. D59 (1999) 055008; O. Brein, W. Hollik and S. Kanemura, Phys. Rev. D63 (2001) 095001; S. Moretti, preprint RAL-TR-2000-038, CERN-TH-2001036, February 2001, hep-ph/0102116; A. Dedes and S. Moretti, proceedings of the '11th Rencontres de Blois: Frontiers of Matter', Chateau de Blois, France, 28 June - 3 July 1999, preprint RAL-TR-1999-067, June 1999, hep-ph/9909526; Eur. Phys. J. C10 (1999) 515; A. Datta, A. Djouadi, M. Guchait and Y. Mambrini, preprint PM/01-26, July 2001, hep-ph/0107271.

[18] M. Drees, M. Guchait and D.P. Roy, Phys. Lett. B471 (1999) 39; S. Moretti, Phys. Lett. B481 (2000) 49.

[19] J. F Gunion and H.E. Haber, Nucl. Phys. B307 (1988) 445; ibidem B272 (1986) 1; Erratum, ibidem B402 (1993) 567; A. Djouadi, P. Janot, J. Kalinowski and P.M. Zerwas, Phys. Lett. B376 (1996) 220.

[20] M. Bisset, M. Guchait and S. Moretti, Eur. Phys. J. C19 (2001) 143.

[21] M. Guchait and S. Moretti, in preparation.

[22] H.L. Lai, J. Huston, S. Kuhlmann, F. Olness, J. Owens, D. Soper, W.K. Tung and H. Weerts, Phys. Rev. D55 (1997) 1280.

[23] D.P. Roy, Phys. Lett. B459 (1999) 607.

[24] CDF Collaboration: F. Abe et al., Phys. Rev. Lett. 79 (1997) 3585.

[25] B.K. Bullock, K. Hagiwara and A.D. Martin, Nucl. Phys. B395 (1993) 499; J.H. Kühn and A. Santamaria, Z. Phys. C48 (1990) 445.

[26] S. Raychaudhuri and D.P. Roy, Phys. Rev. D52 (1995) 1556.

[27] We have used a code provided by D.P. Roy to generate the distributions of the $\tau$-decay products, based on the formalism discussed in [26].

[28] G. Corcella, I.G. Knowles, G. Marchesini, S. Moretti, K. Odagiri, P. Richardson, M.H. Seymour and B.R. Webber, preprint CAVENDISH-HEP-01-08, CERN-TH2001-173, DAMTP-2001-61, July 2001, hep-ph/0107071.

[29] G. Corcella, I.G. Knowles, G. Marchesini, S. Moretti, K. Odagiri, P. Richardson, M.H. Seymour and B.R. Webber, preprint Cavendish-HEP-01/16, CERN-TH/2001369, DAMTP-2001-109 IPPP/01/65, DCPT/01/130, KEK-TH-797, December 2001. 
[30] R. Decker, S. Jadach, M. Jezabek, J.H. Kühn and Z. Was, preprint CERN-TH-6793 (1993); Comp. Phys. Comm. 70 (1992) 69; ibidem 64 (1991) 275; preprint CERNTH-6195 (1991); preprint CERN-TH-5856 (1990). 

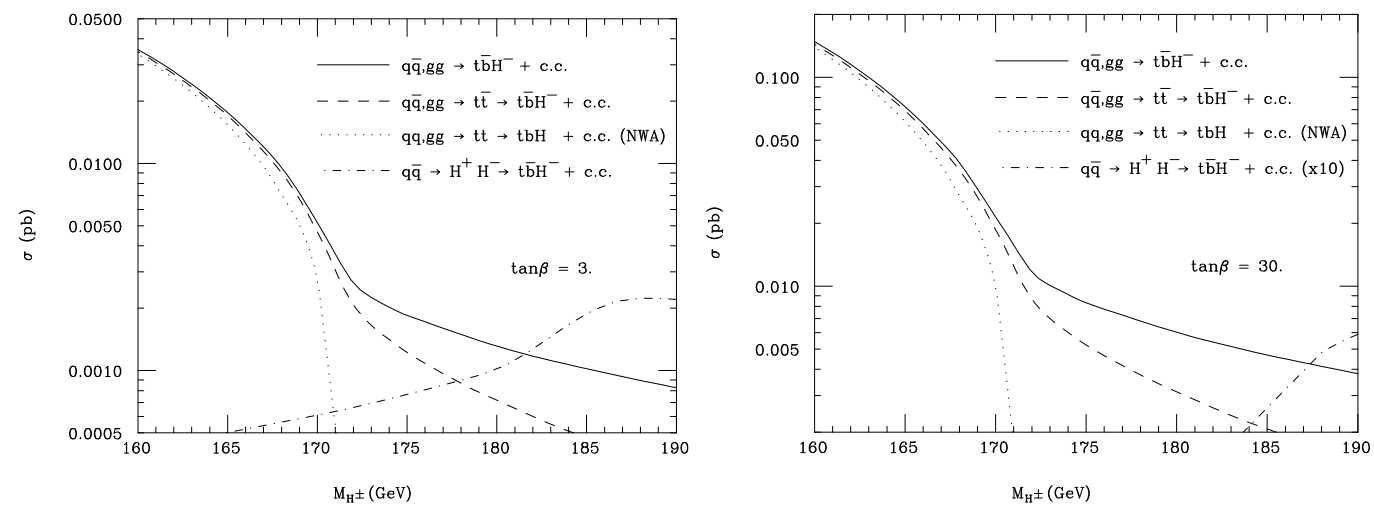

Figure 1: Cross section for $g g, q \bar{q} \rightarrow t \bar{b} H^{-}$(solid), $g g, q \bar{q} \rightarrow t \bar{t} \rightarrow t \bar{b} H^{-}$(dashed, with finite top width) and $g g, q \bar{q} \rightarrow t \bar{t} \rightarrow t \bar{b} H^{-}$(dotted, in NWA), at $\sqrt{s}=2 \mathrm{TeV}$, as a function of $M_{H^{ \pm}}$for two representative values of $\tan \beta$ (hereafter, charge conjugated rates are always included). For comparison, we also have plotted the cross section for $q \bar{q} \rightarrow H^{+} H^{-} \rightarrow t \bar{b} H^{-}$ (dot-dashed). (Notice that the rates for the latter have been multiplied by 10 for the case $\tan \beta=30$, for readability.) 

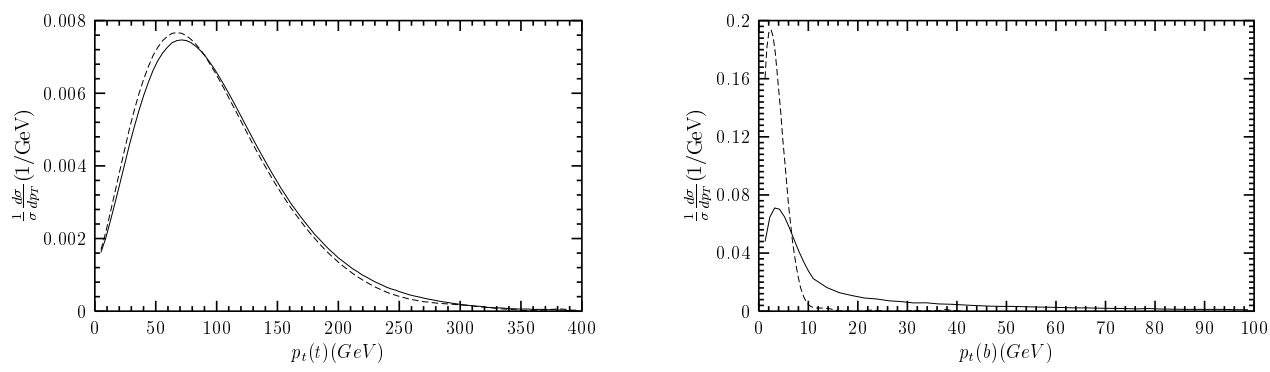

Figure 2: Transverse momentum distributions of the three-body final state top- (left) and bottom-quark (right) in $g g, q \bar{q} \rightarrow t \bar{b} H^{-}$(solid) and $g g, q \bar{q} \rightarrow t \bar{t} \rightarrow t \bar{b} H^{-}$(dashed) (the latter in NWA), at $\sqrt{s}=2 \mathrm{TeV}$, for $M_{H^{ \pm}}=170 \mathrm{GeV}$. (Notice that the spectra are independent of the choice of $\tan \beta$.)
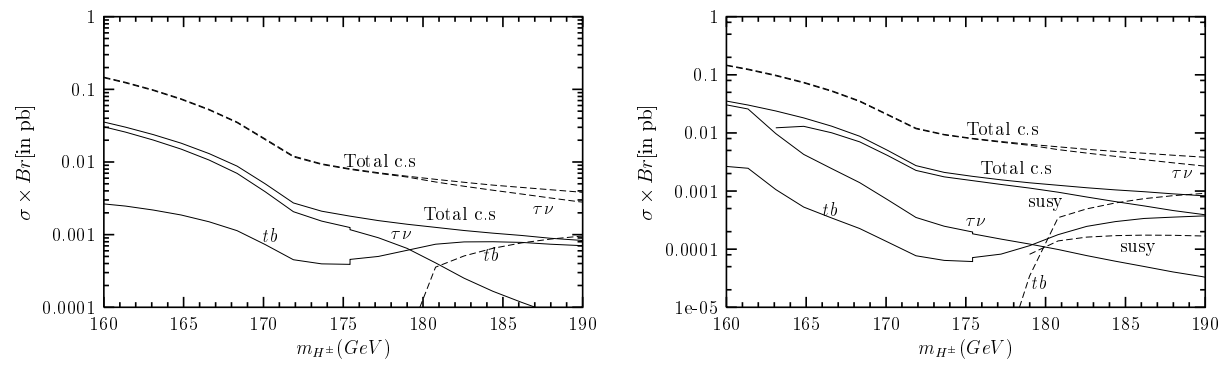

Figure 3: Cross section for $g g, q \bar{q} \rightarrow t \bar{b} H^{-}$times the BRs in all relevant decay modes of charged Higgs bosons, at $\sqrt{s}=2 \mathrm{TeV}$, as a function of $M_{H^{ \pm}}$for $\tan \beta=3$ (solid) and 30 (dashed). On the left-hand side, we assume that decays into Supersymmetric particles are prohibited. On the right-hand side, we include them, by adopting an MSSM setup with $M_{2}=130 \mathrm{GeV}$ and $\mu=300 \mathrm{GeV}$ (see the text for the sparticle masses). 


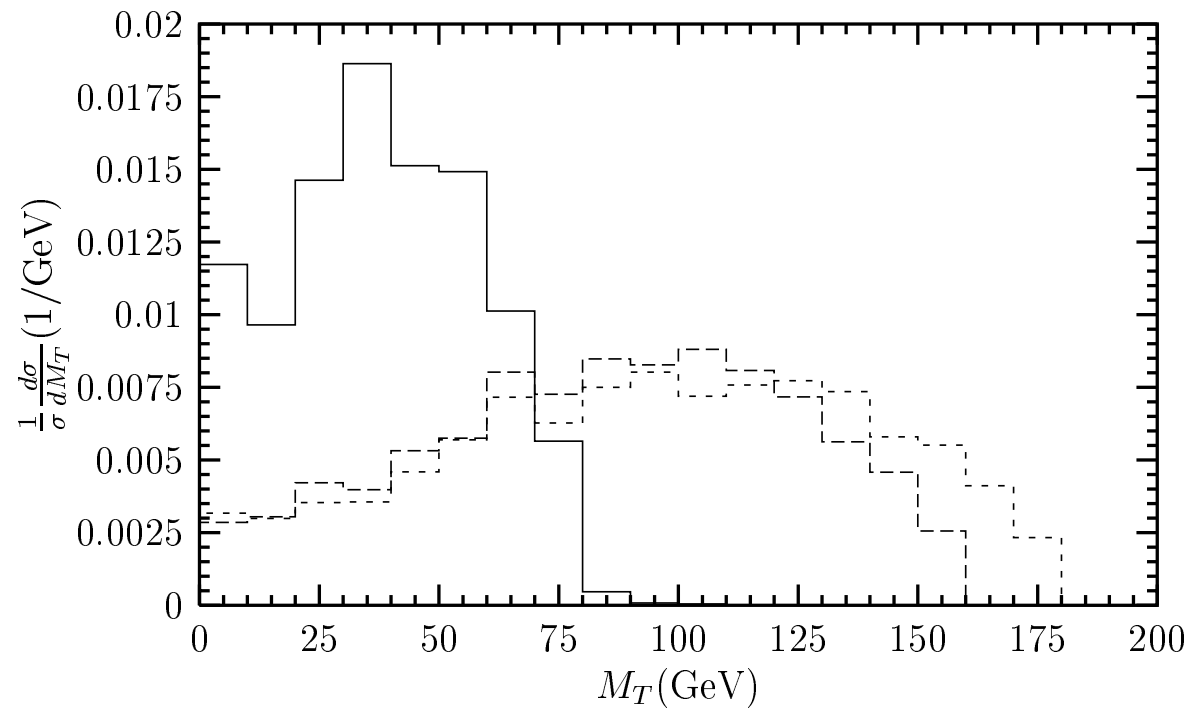

Figure 4: Transverse mass distribution, as defined in eq. (3.1), in $g g, q \bar{q} \rightarrow t \bar{b} H^{-}$, for $M_{H^{ \pm}}=160$ and $180 \mathrm{GeV}$ (long- and short-dashed, respectively), and $g g, q \bar{q} \rightarrow t \bar{b} W^{-}$(solid), after the cuts 1.-4. described in the text. (Notice that the signal spectra are independent of the choice of $\tan \beta$.) 


\begin{tabular}{|c|c|c|c||c|}
\hline$M_{H^{ \pm}}(\mathrm{GeV}) \downarrow / \tan \beta \rightarrow$ & 3 & 6 & 40 & $t \bar{b} W^{-}$ \\
\hline 150 & 6 & 3 & 52 & 0.22 \\
160 & 2.8 & 1.5 & 22 & 0.22 \\
170 & 0.4 & 0.25 & 3.5 & 0.22 \\
175 & 0.13 & 0.08 & 1.42 & 0.22 \\
180 & 0.067 & 0.061 & 1.09 & 0.22 \\
\hline
\end{tabular}

Table 1: The cross section (in fb) for the signal $q \bar{q}, g g \rightarrow t \bar{b} H^{-}\left(\rightarrow \tau^{-} \bar{\nu}_{\tau}\right)$ and the background $q \bar{q}, g g \rightarrow t \bar{b} W^{-}\left(\rightarrow \tau^{-} \bar{\nu}_{\tau}\right)$, at $\sqrt{s}=2 \mathrm{TeV}$, for representative values of $M_{H^{ \pm}}$and $\tan \beta$, after all cuts described in the text. (Notice that the background rates are independent of $M_{H^{ \pm}}$, as the transverse mass constraint that we adopted does not depend on the latter.) 


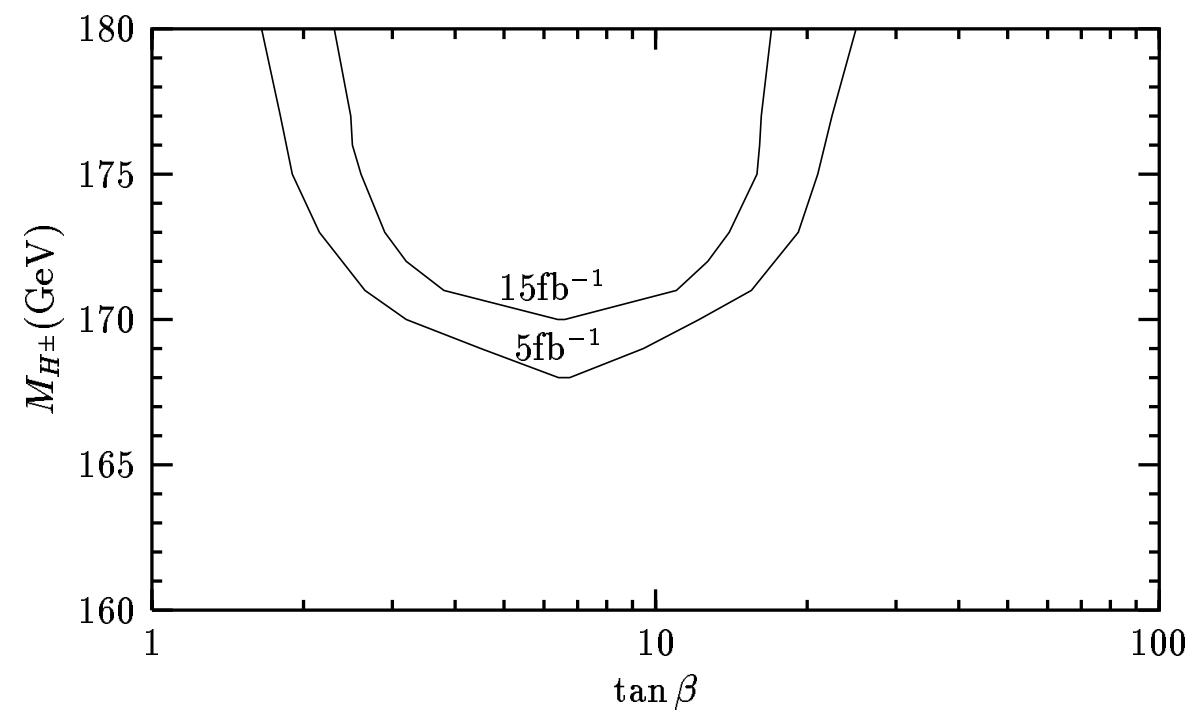

Figure 5: The exclusion regions (below the curves) at 95\% CL in the $M_{H^{ \pm}}-\tan \beta$ plane that can be achieved at Tevatron Run 2 for the two luminosity options of 5 and $15 \mathrm{fb}^{-1}$. 